\title{
Las aptitudes y actitudes empresariales de los estudiantes de Administración en las universidades públicas de Lima - Callao
}

\begin{abstract}
Nemesio Espinoza Herrera"
E-mail: nespinozah@hotmail.com

RESUMEN Objetivos: El estudio plantea como objetivo el de conocer las causas y características del desempleo profesional en el Perú así como de las actuales estrategias educativas vigentes en las facultades de Administración de las universidades públicas y las repercusiones de las actitudes y aptitudes empresariales en los estudiantes de Administracion así como de sus incidencias en el nivel de empleabilidad. Método: El trabajo de campo se ha efectuado en las facultades de Administración de tres universidades públicas ubicadas en las ciudades de Lima y Callao en las que se ha aplicado cuestionarios a estudiantes de diferentes ciclos, edades y turnos de estudio. Resultados: Se aprecia la continuidad -como hacen décadas-de las mismas estrategias formativas orientadas a formar profesionales en Administración para que ocupen cargos gerenciales en las grandes empresas y ante la imposibilidad de tal ocurrencia, los egresados, irremediablemente, conforman un gran contingente de administradores sin empleo. No existen estrategias pedagógicas orientadas a formar empresarios ni a promover aptitudes y actitudes empresariales. Conclusión: La reversión de esta realidad nacional demanda la apremiante necesidad del cambio de paradigmas educativos en las facultades de Administración de las universidades públicas a fin de que estén orientados más a la formación de empresarios y a la inculcación sistemática de las actitudes y aptitudes empresariales en los estudiantes durante su formación profesional.
\end{abstract}

Palabras clave: Formación de empresarios, universidades públicas, desempleo profesional, PYMES.

Entrepreneurial aptitudes and attitudes of management undergraduate students in public universities in the Lima - Callao area

ABSTRACT The objective of this research paper is to know the causes and characteristics of professional unemployment in Perú as well as the current educational strategies in use in management departments of public universities and the aftermath of the entrepreneurial attitudes and aptitudes of management undergraduate students and their incidence in the employment level. Method: The fieldwork on this research has been undertaken in the management departments of three public universities in the cities of Lima and Callao by having students of diverse ages, study years, and study shifts, filling adhoc questionnaires. Results: It has been observed -as with decades ago- the permanence of the same educational strategies oriented towards forming undergraduate professionals in management aspiring to occupy management positions in large and medium size enterprises, and as it is impossible right now, by all means, they integrate a great group of unemployed professional managers. There are neither educational strategies oriented towards educating entrepłeneurs nor to promote entrepreneurial aptitudes and attitudes. Conclusions: In order to invert this Peruvian national reality, it demands the urgent necessity of educational paradigm changes in the undergraduate management departments of public universities in order to be more geared towards the education of entrepreneurs and the systematic deployment of entrepreneurial attitudes and aptitudes on the students during their professional education.

Keywords: Education of entrepreneurs, public universities, professional unemployment, small and micro businesses.

\footnotetext{
- Magister en Administración. Profesor Principal de la Facultad de Ciencias Administrativas de la UNMSM.
} 


\section{INTRODUCCIÓN}

El presente artículo tiene el propósito de cuestionar las estrategias y paradigmas educativos vigentes en las universidades del país, especialmente de las estatales, respecto a la formación de los profesionales en Administración y, por extensión, de otras carreras universitarias. Pretende poner de relieve la importancia de la formación de empresarios en las facultades de Administración y las incidencias en ella de infundir las aptitudes y actitudes empresariales en los estudiantes.

A la luz de los acontecimientos y resultados suscitados en estas últimas décadas, el actual modelo de universidad peruana no responde ante las expectativas y exigencias del desarrollo nacional. El alarmante alto nivel de desempleo profesional existente en el país, constituye un elocuente indicador de los bajos niveles de la calidad académica en la formación de los profesionales y la ausencia de rendimientos en las labores de la Investigación Científica. La universidad peruana, tanto privada como pública, ha quedado a la zaga de los procesos de cambio suscitados en el mundo y, consecuentemente, en el país.

Dos son las misiones fundamentales que por naturaleza innata corresponden a la universidad como concepto y como ente: la formación de profesionales y la producción de la Ciencia y Tecnología a través de la Investigación Científica (Ref. 13). Ambas misiones son pilares básicos para la promoción sostenida del desarrollo económico y social del país. La universidad pública peruana no viene cumpliendo a cabalidad sus misiones con la agravante de que los nuevos contextos de globalización y competitividad exigen de las universidades aportes efectivos traducidos en la dotación de profesionales de nuevo tipo y en la producción científica y tecnológica a través de la Investigación Científica. Estas características peculiares de la universidad peruana traen consigo una consecuencia: el desempleo profesional.

La inserción soberana del país al nuevo contexto de globalización y competitividad mundial, propio del nuevo siglo y milenio, exigen la reconversión de paradigmas educacionales y una reformulación radical -reingeniería- sobre el problema de la universidad y la formación de profesionales de tal forma que contribuyan efectivamente al proceso de desarrollo nacional. La persistencia de los altos índices de desempleo profesional resulta incompatible con las nuevas tendencias mundiales.

En el tema del desempleo (y subempleo) profesional en el Perú, las siguientes aseveraciones confirman con elocuencia la gravedad del problema.
Según estudios realizados (Apoyo S.A., ANR), las carreras universitarias con mayor desempleo profesional son: Educación, Derecho, Ingeniería, Contabilidad y Administración y precisamente en ellas se concentra la mayor oferta (y demanda) universitaria. Se establece niveles del 60 a $75 \%$ de desempleo profesional en el Perú. (Informaciones deducidas de la Refs.: 3, 9, 14, 15 y 16.

"Según datos del INEI, actualmente 59.9\% de los egresados de las universidades no encuentran empleo adecuado en relación con sus estudios" (Goerge Hashaviah en Ref. 15). Según informaciones oficiales egresan de secundaria 400000 , postulan a las universidades 260000 , ingresan 83000 , se titulan 22000 y logra trabajo 5500 . Es decir, $75 \%$ de profesionales ¡sin empleo! Según un estudio del Ministerio de Trabajo, el 71\% de los profesionales terminan haciendo algo distinto de aquello para lo que se prepararon" ( Ref. 8).

En el año 2002 circuló un comercial en el que los egresados universitarios, habiendo hecho esfuerzos en sus estudios, lanzan al cielo sus birretes en la ceremonia de graduación en señal del éxito de ser profesionales; pero, terminan siendo taxistas. Este comercial grafica la realidad universitaria en el Perú y es "un disparo directo a la médula y un mandar al tacho el humor y rescatar la realidad" (Ref. 7). El comercial concluye diciendo "elige bien la universidad" en la que vas ha estudiar. En realidad no se trata de "elegir bien", se trata que el sistema cambie.

Por otra parte, las empresas en general (Ref. 16) constituyen importantes factores para el desarrollo nacional y requieren una administración moderna y eficiente porque los nuevos contextos de globalización y competitividad así lo exigen. Esto explica la vinculación de la empresa con la universidad, más específicamente con las facultades de Administración, por cuanto éstas deben proveer a la sociedad empresarios, administradores o gerentes profesionales probos. Precisamente esta interrelación simbiótica empresa-universidad fundamenta la necesidad de abordar el tema de las actitudes y aptitudes empresariales de los estudiantes de Administración en el marco de la temática de la formación de empresarios en las universidades.

En la actualidad -al igual que las universidades- las empresas en general tienen que ir gradualmente reconvirtiendo sus paradigmas apuntalando hacia el progresivo logro de niveles sostenidos de competitividad cada vez superiores para conquistar mercados nacionales e internacionales. Para ello resulta una absoluta necesidad la existencia de empresarios profesionales de nuevo tipo lo suficientemente formados para hacer frente a los nuevos retos del desarrollo económico y social del país. 
Sin embargo, en el tema de las empresas en general y su vinculación con la formación de empresarios en las universidades, especial atención merece el caso de las pequeñas y microempresas (PYMES) por ser ellas el eje de la economía nacional y mundial. (Las pequeñas y microempresas, según versiones ya muy conocidas en el medio, representan el $99 \%$ de las empresas peruanas, genera el $50 \%$ del PBI y crea empleo en un $75 \%$ de la PEA). La promoción y desarrollo del sector empresarial de las PYMES - una estratégica tarea de política nacional de desarrollo- plantea la imperativa necesidad de gestar una nueva generación de empresarios profesionales de nuevo tipo, exprofesamente formados en las universidades, concretamente en las facultades de Administración; y, consecuentemente, plantea la necesidad de nuevos paradigmas y estilos de Administración o de gestión empresarial.

Esta responsabilidad, sin embargo, no es asumida con plenitud y efectividad por las facul tades de Administración de las universidades por cuanto no forman necesariamente empresarios capaces de gestar PYMES y crear empleo sino, por el contrario, forman profesionales para el desempleo porque al estar sus estrategias educativas y pedagógicas orientadas hacia la formación de gerentes y ejecutivos para el sector empresarial de las grandes empresas -que si bien dinamizan grandes recursos financieros, empero, no generan puestos de trabajo-las universidades forman profesionales que no encuentran oportunidades de empleo.

(En el Perú 22 universidades públicas ofrecen la carrera de Administración con un total de 11568 estudiantes - con datos de la Oficina de Coordinación Universitaria del Ministerio de Educación, ANR y de las universidades correspondientes al segundo semestres 2001 y primer semestre 2002-. En el caso de la Universidad de Huancavelica la carrera de Administración es de reciente creación y se encuentra en implementación. Así mismo se sabe que 31 universidades privadas tienen carreras de Administración).

El tema del desempleo (y subempleo) profesional, a pesar de sus repercusiones negativas en el desarrollo nacional, no es aún materia de estudios e investigaciones científicas. Así mismo, las universidades, principalmente en cuanto se refiere a sus estrategias educativas vigentes, no existen planteamientos de reconversión debido a la carencia de estudios e investigaciones. En cuanto se refiere al tema específico de la formación de administradores profesionales con altos niveles de desempleo, tampoco no ha sido considerado como materia de investigación.

Estas consideraciones han suscitado algunas inquietudes que motivaron la presente investigación: ¿Cuáles son las causas del creciente desempleo profesional en Administración y, por extensión, de las carreras profesionales en el Perú? ¿Cuál es la influencia de la formación de empresarios en las universidades y, dentro de ella, de la inculcación de las actitudes y aptitudes empresariales en los estudiantes de Administración? ¿Qué repercusiones tienen ellas en el nivel de empleabilidad de los egresados universitarios? ¿Cuáles son las características de las actuales estrategias formativas en las facultades de Administración? ¿Cuáles son los elementos ejes a tomarse en cuenta en un proceso de reconversión universitaria que posibilite la disminución gradual pero sostenida del desempleo profesional en el Perú?

\section{MÉTODO}

El trabajo de campo se ha efectuado en las facultades de Administración de tres universidades públicas ubicadas en las ciudades de Lima y Callao: La Universidad Nacional Mayor de San Marcos, la Universidad Nacional Federico Villarreal y la Universidad Nacional del Callao. En estas universidades se ha aplicado un total de 200 cuestionarios a igual número de estudiantes de diferentes ciclos, edades y turnos de estudio. En cuanto a la Universidad Nacional Faustino Sánchez Carrión de Huacho -inicialmente considerada como parte de la muestra- por razones de la huelga nacional indefinida de docentes, no se ha logrado realizar las encuestas, aunque se ha efectuado las coordinaciones preliminares para una entrevista con el decano de la Facultad de Administración (Lic. Luis Baldeón Ardián).

Téngase en cuenta que por razones ajenas a la voluntad de los investigadores -huelga de docentes, estudio sin apoyo económico para su ejecución, etc.no se ha logrado encuestar a mayor número de estudiantes. De la Universidad de San Marcos se ha encuestado a 127 estudiantes, de la Universidad del Callao 58 y de Federico Villarreal 15. Estas eventualidades corroboran la necesidad de emprender nuevos estudios de mayor envergadura sobre el tema que al tener una mayor cobertura, inclusive nacional, sus resultados sean mucho más representativas.

También se ha efectuado entrevistas en profundidad a las autoridades, docentes y estudiantes de las universidades señaladas. 


\section{RESULTADOS}

TABLA N. ${ }^{\circ} 1$

200 ENTREVISTAS

\begin{tabular}{|c|c|c|c|c|c|c|c|c|c|c|}
\hline & \multicolumn{2}{|c|}{$\begin{array}{c}\text { EDAD } \\
\text { DE LOS } \\
\text { ESTUDIANTES } \\
\text { ENCUESTADOS }\end{array}$} & \multicolumn{2}{|c|}{ TURNO } & \multicolumn{2}{|c|}{$\begin{array}{c}\text { LUGAR DE } \\
\text { NACIMIENTO }\end{array}$} & \multicolumn{2}{|c|}{$\begin{array}{l}\text { ESTUDIANTES } \\
\text { CON TRABAJO } \\
\text { DEPENDIENTE }\end{array}$} & \multicolumn{2}{|c|}{$\begin{array}{l}\text { ESTUDIANTES } \\
\text { QUE NO } \\
\text { TRABAJAN } \\
\text { PERO TIENEN } \\
\text { NEGOCIO }\end{array}$} \\
\hline & 19-23 & $24-25$ & DÍA & NOCHE & LIMA & PROVINCIA & SÍ & NO & SÍ & NO \\
\hline CANT. & 160 & 40 & 105 & 95 & 160 & 40 & 87 & 113 & 19 & 94 \\
\hline$\%$ & 80.0 & 20.0 & 52.5 & 47.5 & 80.0 & 20.0 & $\begin{array}{c}43 . \\
5\end{array}$ & $\begin{array}{c}56 . \\
5\end{array}$ & $\begin{array}{c}16 . \\
8\end{array}$ & 83.2 \\
\hline
\end{tabular}

TABLA $\mathbf{N}^{\circ} 2$

200 ENTREVISTAS

\begin{tabular}{|c|c|c|c|c|c|c|c|c|c|c|c|c|c|c|c|c|c|}
\hline \multicolumn{4}{|c|}{$\begin{array}{c}\text { FINANCIAMIENTO } \\
\text { DE ESTUDIANTES } \\
\text { QUE NO TRABAJAN } \\
\text { Y NO TIENEN } \\
\text { NEGOCIO PROPIO }\end{array}$} & \multicolumn{5}{|c|}{$\begin{array}{c}\text { OBJETIVOS DE LOS } \\
\text { ESTUDIANTES QUE NO } \\
\text { TRABAJAN Y NO TIENEN } \\
\text { NEGOCIO PROPIO }\end{array}$} & \multicolumn{6}{|c|}{$\begin{array}{l}\text { RAZONES POR LAS QUE NO } \\
\text { INCURSIONAN EN NEGOCIOS } \\
\text { AQUELLOS QUE TRABAJAN } \\
\text { PARA OTROS Y LOS QUE NO } \\
\text { TIENEN NEGOCIOS }\end{array}$} & \multicolumn{3}{|c|}{$\begin{array}{l}\text { GENERAL } \\
\text { SOBRE SI EL } \\
\text { EMPRESARIO } \\
\text { NACE O SE } \\
\text { HACE }\end{array}$} \\
\hline 1 & 2 & 3 & 4 & 5 & 6 & 7 & 8 & 9 & 10 & 11 & 12 & 13 & 14 & 15 & 16 & 17 & 18 \\
\hline 76 & 7 & 8 & 3 & 16 & 26 & 24 & 25 & 3 & 5 & 16 & 29 & 77 & 46 & 8 & 32 & 164 & 4 \\
\hline 80.9 & 7.4 & 8.5 & 3.2 & 17.0 & 27.7 & 25.6 & 26.6 & 3.2 & 2.8 & 8.8 & 16.0 & 42.5 & 25.4 & 4.4 & 16.0 & 82.0 & 2.0 \\
\hline
\end{tabular}

1. Padres, 2. Familiares, 3. Con trabajo esporádico, 4. Beca, 5. Busco trabajo, 6. Concluir estudios, 7. Crear un negocio 8. Hacerprácticas, 9. Otro, 10. No estoy interesado, 11. No tengo experiencia, 12. Lo haré cuando egrese, 13. No tengo capital, 14. Estoy elaborando un proyecto, 15. Estoy buscando financiamiento, 16. Nace, 17. Se hace, 18. Nace y se hace.

\section{CARACTERÍSTICAS GENERALES DE LOS ENCUESTADOS}

El $52.5 \%$ de Ios encuestados estudian en el turno diurno, mientras que el $47.5 \%$ en el turno nocturno. El $80 \%$ han nacido en la región Lima y el $20 \%$ en provincias. Los estudiantes encuestados pertenecen al grupo comprendido entre el sexto y décimo ciclo. El $80 \%$ de ellos tienen edades que fluctúan entre 19 a 23 años y $20 \%$ entre 24 a 25 .

El hecho de que casi la mitad de los estudiantes se encuentren matriculados en el turno nocturno significa que una gran parte del tiempo de estos estudiantes se insume en preocupaciones laborales (unos buscando el sustento económico, otros inmerso en el subempleo o desempleo). Sea cualquiera fuera el caso, lo cierto es que profesionales formados sólo a tiempo parcial y sólo en horas marginales no es garantía de la calidad académica y de la sólida formación profesional.

\section{SITUACIÓN LABORAL DE LOS ESTUDIAN- TES DE ADMINISTRACIÓN}

El $43.5 \%$ de los encuestados declaran trabajar de manera dependiente. El 9.5\% declaran tener negocio propio. Esto quiere decir que el $47 \%$ de los estudiantes sólo se dedican a sus estudios.

Este da to corrobora el comentario anterior, en el sentido de que casi la mitad de los estudiantes trabajan en forma dependiente. Si se asume un promedio de 8 horas de jornada laboral mínima, 8 horas de descanso y cuatro horas dedicados a traslados, restarían 4 escasas horas para sus estudios absolutamente insuficientes para una sólida formación. Esta realidad explica la baja calidad formativa en la que las investigaciones científicas se encuentran ajenas y explica, consecuentemente, el bajo nivel de empleabilidad de sus egresados. 


\section{RESPECTO A LOS ESTUDIANTES QUE DE- CLARAN TENER NEGOCIO PROPIO}

De los 113 estudiantes encuestados (56.5\%) que declaran no trabajar de manera dependiente, el $16.81 \%$ declaran tener negocio propio y el $83.19 \%$ declaran no tenerlo. Precisa reiterar que de la totalidad de los encuestados sólo el $9.5 \%$ declaran tener negocio propio.

Esto quiere decir, en términos generales, que de cada 100 estudiantes de Administración, 10 tienen su propio negocio, sobre todo en el sector de los que cursan los últimos ciclos. Al respecto precisa comentar que estos resultados no siempre reflejan la realidad porque las respuestas frente a la interrogante si tienen o no negocio propio, al responder positivamente casi siempre se refieren a negocios de sus padres o familiares en los que trabaja o se refieren a pequeños negocios esporádicos de tipo ambulatorio o marginal. Si estas últimas consideraciones resultaran ciertas, entonces se podría deducir que en las facultades de Administración de las universidades públicas los estudiantes no gestan empresas o negocios.

Esta realidad -aun cuando en el hipotético caso fuera el $10 \%$ de estudiantes con negocio propio-redunda preocupación por cuanto demuestra que las estrategias formativas en las facultades de Administración no están orientadas hacia el emprendedorismo. ("El emprendedorismo o empresarialidad es la actitud o espíritu emprendedor y que en inglés se denomina entrepreneurship" (Ref. 5). Una facultad que apunte sus estrategias pedagógicas hacia el empresariado, significaría que la mayor parte de los estudiantes -en especial de los últimos ciclos-ya hayan puesto en marcha sus proyectos empresariales.

(Es importante anotar la necesidad de que existan mecanismos que posibiliten que los egresados de Administración, habiendo obtenido el grado académico de Bachiller en Administración necesariamente mediante la presentación y sustentación de tesis, obtengan el título profesional de Licenciado en Administración a través de la demostración de la viabilidad de funcionamiento de un proyecto empresarial. Esto permitiría titular a los egresados sólo si demuestran que pueden crear y geștionar una empresa).

Este tema -estudiantes de Administración con pequeñas o microempresas propias-constituye un tema potencial de investigación de mucha importancia y prioritaria por cuanto sus resultados permitirán conocer las repercusiones de la formación de empresarios en las universidades.

\section{SOBRE QUIENES ASUMEN LOS GASTOS DE ESTUDIOS DE LOS ESTUDIANTES DE ADMINISTRACIÓN QUE NO TRABAJAN NI TIENEN NEGOCIO PROPIO}

Del total de encuestados, 87 (43.5\%) declaran tener trabajo dependiente, $19(9.5 \%)$ declaran tener negocio propio. Esto quiere decir que los estudiantes que dependen de otras personas para solventar sus gastos son $106(47 \%)$.

En la población estudiantil que no trabajan ni tienen negocio propio, en el $80.85 \%$ de los casos son sus padres quienes asumen los gastos de estudios. Un $7.44 \%$ declaran que son sus familiares. El $8.52 \%$ declaran solventar sus gastos con pequeños trabajos esporádicos y el 3.19\% declaran tener becas de estudios.

En el grueso sector de estudiantes que dependen integralmente de sus padres (o familiares directos) se observa que no es usual que ellos estén pensando y actuando en la posibilidad de crear sus propios negocios o empresas, cuando debían hacerlo. Al no tener preocupaciones de orden económico para su manutención y para sus estudios, en lugar de ir creando condiciones para incursionar en la actividad empresarial, están esperando - equivocada conjetura- terminar sus estudios para "encontrar" un puesto de trabajo en las empresas; hecho que es más probable que no ocurra.

\section{OBJETIVO ACTUAL DE LOS ESTUDIANTES DE ADMINISTRACIÓN QUENO TRABAJAN NI TIENEN NEGOCIO PROPIO}

Siendo el $47 \%$ de los encuestados (94) los que no trabajan de manera dependiente ni tampoco tienen negocio propio, consultados sobre sus objetivos inmediatos el $27.65 \%$ respondieron que es el concluir sus estudios y trabajar. El $26.59 \%$ respondieron que es hacer prácticas profesionales, el $25.55 \%$ crear negocio propio, el $17.02 \%$ buscar trabajo y otro el $3.19 \%$ (salir al extranjero).

Dicho y hecho. La mayor parte de los estudiantes están esperando (con ansias aunque con pasividad) concluir sus estudios para "buscar" trabajo. Sin embargo, ocurre que obtenido el grado, el título, inclusive postgrado -siendo la realidad distinta a la que la describieron en la universidad-no encuentran puestos para los cuales han sido formados durante varios años.

Resulta de mucha importancia, por lo tanto, la necesidad de hacer un seguimiento de los egresados (con grados y títulos). Este es otro tema de investigación cuyos resultados nos permitirían 
conocer a ciencia cierta de qué están haciendo los egresados de Administración de las universidades.

El hecho de que un buen sector de estudiantes tenga como objetivo inmediato el hacer prácticas profesionales resulta también algo que refleja la problemática. Las prácticas preprofesionales, en realidad, en vez de ser objetivo de los estudiantes debía ser parte de la estrategia formativa de las facultades. Es responsabilidad de ellas incluir el tema de las prácticas profesionales en la estructura curricular como parte de la formación profesional. Empero, al no existir estrategias educativas que apunten a la fusión de la teoría (en las aulas) con la práctica (en las entidades) desde el primer ciclo mismo; los egresados están distanciados de la realidad. Esto porque, entre otras cosas, no existe la vinculación empresa-universidad.

Una nueva concepción de la formación de los profesionales en Administración implicará necesariamente que para la existencia de una Facultad de Administración sea prerrequisito indispensable mostrar con qué empresas y/o instituciones (o conglomerados, o gremios) están vinculadas formalmente.

\section{RAZONES DE POR QUÉ LOS ESTUDIANTES QUE TRABAJAN DE MANERA DEPENDIEN- TE $Y$ LOS QUE NO TRABAJAN NO INCURSIONAN EN LOS NEGOCIOS}

Del total de 181 encuestados que sí trabajan de manera dependiente y no tienen trabajo, argumentaron frente a la interrogante de la razón de por qué no incursiona en alguna actividad empresarial respondieron: el $42.54 \%$ porque no tiene capital, el $25.42 \%$ porque recién está elaborando un proyecto de negocio, el $16.03 \%$ responde que lo hará cuando egrese o concluya su profesión, el $8.83 \%$ respondieron porque no tienen experiencia, el $4.42 \%$ que está buscando financiamiento, el $2.76 \%$ porque no está interesado en hacer empresa. Las alternativas de "no tengo vocación de empresario" y la de "otro" no tuvieron respuestas.

\section{OPINIONES SOBRE EL EMPRESARIADO DE LOS ESTUDIANTES DE ADMINISTRA- CIÓN}

Preguntados al $100 \%$ de los encuestados sobre si el empresario nacía o se hacía (formación de empresarios), el $82 \%$ respondieron que el empresario se hace, el $16 \%$ que el empresario nace y un $2 \%$ respondieron que nace y se hace.

Sobre si las facultades de Administración debían formar empresarios, la totalidad de los encuestados respondieron que sí. Interrogados sobre si creen que las facultades de Administración están formando empresarios, el $51.5 \%$ respondieron que sí y el $48.5 \%$ que no.

\section{DISCUSIÓN}

La reconversión de las facultades de Administración de las universidades públicas implica la adopción de nuevas estrategias competitivas que vayan desde una reestructuración radical de sus currículos que deben estar orientados prioritariamente hacia al empresariado, pasando por admitir sólo a estudiantes con probada vocación empresarial y de liderazgo, hasta al logro de egresados con niveles adecuados de empleabilidad, con plenas capacidades profesionales para crear empresas, especialmente del sector de las pequeñas y microempresas.

En las facultades de Administración de las universidades públicas del Perú, siguen vigentes estrategias educativas arcaicas cuyos efectos se expresan en la formación de administradores para el desempleo. No existe tampoco voluntades ni indicios de cambio. Se observa la inexistencia en estas facultades de estrategias formativas de empresarios. Los estudiantes, como es de esperarse, no están mentalizados para hacer empresa sino para ser gerentes o ejecutivos de grandes empresas corroborándose la conjetura de que en las facultades de Administración de las universidades públicas se forman profesionales con "mentalidad de empleado".

Es importante destacar que el estudio muestrano sólo por el aspecto cuantitativo de las respuestas a los cuestionarios aplicados sino por las observaciones y experiencias directas de los investigadores especialmente en cuanto a estrategias pedagógicas (currículos, por ejemplo) se refiere, así como por las entrevistas en profundidad efectuadas-que en las universidades públicas se siguen formando profesionales para el desempleo y no existe estrategias pedagógicas orientadas hacia la selección vocacional y formación de empresarios.

Por lo tanto, el estudio plantea la imperativa necesidad del cambio de estrategias educativas y formativas en las facultades de Administración -en términos de reingeniería pedagógica y curricularde las universidades estatales, las mismas que deben estar orientadas principalmente a la identificación de estudiantes con vocación empresarial y a la formación de empresarios y líderes antes que la dogmática formación dirigida a formar profesionales para ocupar puestos de trabajo inexistentes. De esta forma se espera la gradual pero sostenida disminución del desempleo profesional en Administración en el país. 
Por lo manifestado, y ante el grave problema del desempleo profesional en general y en particular del desempleo profesional en Administración, se formula las siguientes propuestas:

a. Prohibición inmediata de la creación de nuevas universidades públicas y, consecuentemente, de nuevas facultades de Administración.

b. Alianza estratégica entre todas las facultades de Administración de las universidades públicas del país (conformación de un consorcio) para:

1. Ejecución de Investigaciones sobre la carrera profesional de Administración (realidad nacional, diagnósticos, seguimientos de egresados, currículos, empleo y desempleo, etc.).

2. Formación de una entidad autónoma de acreditación universitaria de las facultades de Administración.

3. Elaboración de una nueva política y estrategia nacional de formación de los profesionales en Administración.

c. Reducción drástica de vacantes en las facultades de Administración en las universidades públicas por lo menos a la mitad. Aulas diseñadas con capacidades para 30 ó 40 estudiantes como máximo y provistas necesariamente de un proyector multimedia, equipo de vídeo y pizarras acrílicas (a fin de evitar la excesiva masificación estudiantil que acarrea mediocridad académica). Son preferibles pocos estudiantes universitarios en Administración pero bien preparados de tal modo que cuando egresen contribuyan al desarrollo del país (teniendo empleo y /o generando riqueza y puestos de trabajo como empresarios) a tener profesionales en Administración desempleados en masa.

d. Establecimiento de nuevos mecanismos de admisión a las carreras profesionales de Administración que garanticen que a ellas ingresen sólo aquellos estudiantes de probada capacidad vocacional de liderazgo, de talento empresarial, así como de vocación hacia el trabajo académico universitaria y hacia la Investigación Científica. Esto evitará que en las facultades de Administración de las universidades públicas exista una enorme cantidad de estudiantes sin vocación de liderazgo, sin vocación empresarial y sin capacidades para el riguroso trabajo universitario ni para realizar investigaciones científicas mucho menos. Esa gran mayoría de estudiantes están ahí no por vocación sino porque no hay dónde estar. Una vez más, hoy las universidades públicas se constituyen en refugio del desempleo juvenil.

e. Reglamentos académicos rigurosos en las universidades públicas que permitan una evaluación permanente de los rendimientos a fin de separar a aquellos estudiantes que no responden a las exigencias académicas y científicas de una nueva facultad de Administración.

f. Implementación obligatoria de programas de Estudios Generales en todas las facultades de Administración. Ocurre que hoy egresan universitarios casi analfabetos respecto a la cultura y conocimientos universales y, por lo tanto, están privados de elementos de juicios necesarios para su mejor desenvolvimiento profesional.

g. Una reformulación de la docencia universitaria en Administración basadas en una sólida formación en cuanto a la pedagogía universitaria. Consecuentemente, creación de programas de pedagogía universitaria en Administración en la unidad de postgrado en Administración y la exigencia de iniciar la carrera de la docencia universitaria con el grado académico de maestro.

h. Específicamente en cuanto a la formación de los empresarios en las facultades de Administración así como a la promoción de las actitudes y aptitudes empresariales de los estudiantes se propone:

1. Priorizar en las facultades de Administración de las universidades públicas, la estrategia educativa de la formación de empresarios antes que la tradicional estrategia de formar "ejecutivos" o "gerentes" para ocupar cargos gerenciales en las empresas. Para ello será necesario la implementación de investigaciones sobre las características de la formación de empresarios a fin de formular políticas y estrategias educativas convenientes e implementarlas a partir de los primeros ciclos. Esto implica que en el diseño curricular se establezca una línea troncal prioritaria para la formación de empresarios.

2. Establecer como requisito indispensable para el funcionamiento de una facultad de Administración la de estar vinculada formalmente al sector empresarial, en especial a los conglomerados de las PYMES, a fin de que tanto estudiantes como docentes 
realicen sus labores académicas en estrecha relación con la práctica empresarial desde los primeros ciclos.

3. Promoción de las investigaciones científicas en el ámbito empresarial, a partir del primer ciclo y durante su formación profesional, principalmente orientadas a las innovaciones y transferencias tecnológicas.

4. Reivindicación de la elaboración y sustentación de tesis o de proyectos empresariales viables para optar los grados y títulos.

5. Establecer un sistema de motivación para que los estudiantes desde sus primero ciclos vayan orientado sus esfuerzos hacia la creación de PYMES (concursos, financiamientos, cuadros de méritos, premios anuales a los estudiantes empresarios, ferias, etc.).

6. Generación de un Fondo para el capital semilla a fin de financiar y asesorar a la elaboración de proyectos empresariales.

7. Conformación de un sistema de incubadoras empresariales como fase inicial para la conformación de los parques tecnológicos.

\section{AGRADECIMIENTOS}

Especial agradecimiento a los alumnos, docentes y autoridades de la Facultad de Ciencias Administrativas de las Universidad Nacional Mayor de San Marcos con quienes hemos compartido opiniones y criterios sobre el problema. Agradecimiento especial al Lic. Feliciano Oncevay Espinoza, Decano de la Facultad de Administración de la Universidad Nacional Federico Villarreal y al Lic. Carlos Diez Arenas, Director de la Escuela de Administración de la Universidad Nacional del Callao, quienes nos han atendido y ofrecido sus valiosas opiniones sobre el tema del desempleo profesional en Administración y sobre las repercusiones en él de la formación de las capacidades empresariales en los estudiantes. Igualmente el agradecimiento a los docentes y alumnos de las facultades referidas que nos apoyaron en la realización de las encuestas.

\section{REFERENCIAS}

1. “¿Existe subempleo profesional en el Perú urbano?" En Investigaciones Breves N. ${ }^{\circ}$ 17. Cybele
Burga, Martín Moreno, 2001. GRADE (Grupo de Análisis para el Desarrollo).

2. Análisis dinámico del desempleo en el Perú (Un). Juan Chacaltana. INEI, 2000.

3. Censo Económico Nacional 1994. Instituto Nacional de Estadística e Informática (INEI).

4. Censo Nacional Universitario 1996 (I). Convenio ANR-INEI. 1997.

5. "Condiciones para desarrollar el espíritu empresarial". Fernando Villarán en un Boletín de ESAN, agosto 2002.

6. Conversatorio sobre la universidad peruana. Congreso de la República. 1998.

7. Diario El Comercio, 29/09/02.

8. Diario PERÚ21, 19/02/03.

9. Estudio del perfil del mercado educativo. APOYO Opinión y Mercado S.A. Junio 1999 con actualizaciones al 2001.

10. "Formación de empresarios en las universidades" (La). Nemesio Espinoza Herrera. Artículo en la revista Gestión en el Tercer Milenio. Facultad de Ciencias Administrativas de la UNMSM. Lima, 2000.

11. Gerencia Universitaria, Consideraciones para una nueva Administración Universitaria. Nemesio Espinoza. Editorial San Marcos. Lima, 2000.

12. Ley Universitaria N. ${ }^{\circ} 23733.1983$.

13. Misión de la Universidad. José Ortega y Gasset. Colección el Arquero, Editorial Revista de Occidente, Madrid, 1968.

14. Páginas Web de: Universidades, ANR, Universia, Ministerio de Trabajo, Congreso de la República, Grade, Apoyo S.A. (tips estadísticos).

15. Pequeña y microempresa en el desarrollo nacional (La). Congreso de la República. Lima, mayo de 1998.

16. Perú: the top 10000 companies 2002 (y version anterior 1998). The Perú Report.

17. Profesionales en el tratado bilateral y el ALCA(Los). Red Colombiana de Acción frente al Libre Comercio y el ALCA. Octubre de 2003.

18. Subempleo profesional en el Perú: Un análisis de la situación de los egresados de educación superior universitaria. Proyecto de Estudio.- Martín Moreno. Apoyo S.A. 1999. 